\title{
Genetic variants in microRNAs predict non-small cell lung cancer prognosis in Chinese female population in a prospective cohort study
}

\author{
Xia Lingzi ${ }^{1,2}$, Yin Zhihua ${ }^{1,2}$, Li Xuelian ${ }^{1,2}$, Ren Yangwu ${ }^{1,2}$, Zhang Haibo ${ }^{3}$, Zhao Yuxia ${ }^{4}$, \\ Zhou Baosen ${ }^{1,2}$ \\ ${ }^{1}$ Department of Epidemiology, China Medical University, Shenyang, Liaoning, 110122, Peoples R China \\ ${ }^{2}$ Key Laboratory of Cancer Etiology and Prevention, China Medical University, Liaoning Province Department of Education, \\ Shenyang, Liaoning, 110122, Peoples R China \\ ${ }^{3}$ Department of Radiotherapy, Shenyang North Hospital, Shenyang, Liaoning, 110001, Peoples R China \\ ${ }^{4}$ Department of Radiotherapy Oncology, The Fourth Affiliated Hospital of China Medical University, Shenyang, Liaoning, \\ 110001, Peoples R China
}

Correspondence to: Zhou Baosen, email: bszhou@mail.cmu.edu.cn

Keywords: miRNA, SNP, lung cancer, prognosis, Chinese

Received: March 22, 2016

Accepted: October 17, 2016

Published: November 04, 2016

\section{ABSTRACT}

To investigate the prognostic effect of microRNA single nucleotide polymorphisms (SNP) on non-small cell lung cancer (NSCLC) patients, 658 female participants from northeast China were enrolled in our prospective cohort study and followed up from 2010 to 2015. C-containing genotypes of miR-149 rs2292832 were associated with better overall survival (OS). The joint effect of miR-149 and miR-196a2 and the joint effect of miR-149 and miR-608 were also observed in our study. To verify the function of miR-149 rs2292832, A549 cell lines were stably transfected with lenti-virus containing miR-149-C vector, miR-149-T vector and empty vector. Cells containing $C$ allele assumed a higher expression level of miR-149, a decrease in cell growth and the sensitivity to anticancer drug when compared with cells containing T allele. The role of miR-149 playing in cancer prognosis may function through DNA topoisomerases 1 (TOP1) pathway, according to the results from luciferase reporter assays. In conclusion, miR-149 $\mathrm{C}$ allele may be a prognostic biomarker for better NSCLC OS.

\section{INTRODUCTION}

Lung cancer is a common malignancy worldwide, accounting for $23.6 \%$ deaths due to cancers. In China, the mortality of lung cancer is the highest for both men and women $[1,2]$. The overall 5 -year-survival is only $16.1 \%$ for Chinese female population as reported in a recent cohort study [3]. The poor survival rates for female lung cancer patients call for the refinements in clinical treatment.

Recently, microRNA has emerged as an influential factor in lung cancer progression [4]. microRNAs are a class of 19-25nt in length, small non-coding RNAs [5]. Aberrant expression profiles and genetic polymorphisms of some microRNAs have been shown to be associated with cancer survival [6-9].
Genetic variants presented in miRNA genes or genes being involved in processing mechanisms may inhibit miRNA expression [10]. Moreover, SNPs located in some specified sites may weaken the affinity between miRNAs and their target mRNAs [11]. In our present study, we aimed to investigate the effects of genetic polymorphisms in pre-miRNAs on the prognosis of non-small cell lung cancer.

\section{RESULTS}

No statistical significance was observed for baseline characteristics between the lost to follow-up group and the follow-up group (Supplementary Table S1). The characteristics of the follow-up group were listed in Table 1. As shown in the table, the significant death 
Table 1: The baseline characteristics of the non-small cell lung cancer patients

\begin{tabular}{|c|c|c|c|c|c|c|c|}
\hline Characteristics & $\begin{array}{l}\text { cases } \\
(584) \\
\end{array}$ & $\%$ & Deaths & $\%$ & P-value & MST (months) & $\begin{array}{c}\text { Log-rank } \\
\text { P-value }\end{array}$ \\
\hline Age(yr) & & & & & 0.579 & & 0.856 \\
\hline$\leq 60$ & 252 & 43.1 & 209 & 82.9 & & 25.54 & \\
\hline$>60$ & 332 & 56.9 & 281 & 84.6 & & 25.86 & \\
\hline \multicolumn{8}{|l|}{ Tobaccon exposure } \\
\hline Smoker & 326 & 55.8 & 273 & 83.8 & 0.613 & 22.90 & 0.146 \\
\hline Non-smoker & 258 & 44.2 & 220 & 85.2 & & 18.53 & \\
\hline Histological type & & & & & 0.406 & & $<0.001$ \\
\hline $\mathrm{AD}$ & 317 & 54.3 & 260 & 82.0 & & 22.79 & \\
\hline SQU & 230 & 39.4 & 201 & 87.4 & & 25.01 & \\
\hline others & 37 & 6.3 & 29 & 78.4 & & 34.80 & \\
\hline Clinical stage & & & & & $<0.001$ & & $<0.001$ \\
\hline I & 132 & 22.6 & 94 & 71.2 & & 41.56 & \\
\hline II & 65 & 11.1 & 51 & 78.5 & & 27.43 & \\
\hline III & 309 & 52.9 & 275 & 89.0 & & 18.86 & \\
\hline IV & 78 & 13.4 & 70 & 89.7 & & 23.67 & \\
\hline Chemotherapy & & & & & 0.225 & & $<0.001$ \\
\hline no & 55 & 9.4 & 43 & 78.2 & & 15.15 & \\
\hline yes & 529 & 90.6 & 447 & 84.5 & & 27.67 & \\
\hline Surgery & & & & & 0.218 & & 0.005 \\
\hline no & 200 & 34.2 & 173 & 86.5 & & 22.48 & \\
\hline yes & 384 & 65.8 & 317 & 82.6 & & 27.40 & \\
\hline
\end{tabular}

*AD: adenocarcinoma, SQU:squamous carcinoma, others: large cell carcinoma or mixed types, MST: median survival time.

rate was observed only in patients with different clinical stages $(\mathrm{P}<0.001)$. While, the significant median survival time (MST) can be observed in patients with different histological types, clinical stages and in patients with or without receipt of chemotherapy or surgery $(\mathrm{P}<0.05)$.

The association between the five SNPs and OS was summarized in Table 2. Patients containing $\mathrm{C}$ allele of miR-149 rs2292832 may have a better OS, longer MST and lower death rate. The effect of miR-149 SNPs on survival rate may be associated with the number of $\mathrm{C}$ alleles. Patients with more $\mathrm{C}$ allele may have a better prognosis. Figure 1 was the survival curves for patients with different genoytypes of miR-149 rs2292832. No statistically significant association was observed for the other four SNPs.

Given the effect of patients baseline characteristics on survival, we proceeded to conduct stratified analyses. Results were summarized in Table 3. The protective role of C-containing genotypes of miR-149 were widely observed in patients from different strata. Figure 2 was the survival curves for I/II/III patients with different genotypes. The protective effect of $\mathrm{CC}$ genotypes in miR-196a2 rs11614913 was observed in patients with squamous carcinoma. CC genotype of miR-608 rs4919510 may function as a risk factor for patients receiving chemotherapy or surgery. No significant association was observed for miR-146a and miR-423 polymorphisms in the stratified analyses (data not shown).

The joint effect for the miRNA SNPs was estimated in our study. Results were summarized in Table 4. As shown in the table, the joint effect of miR-149 SNP and miR-196a2 SNP appeared in patients with squamous cell carcinoma. The joint effect of miR-149 SNP and miR608 SNP appeared in patients receiving chemotherapy or surgery.

To explore the impact of these five SNPs on expression levels in lung cancer tissues, we conducted the qRT-PCR and results were summarized in Supplementary 
Table 2: The association between the five SNPs and OS

\begin{tabular}{lccccccc}
\hline $\begin{array}{l}\text { SNP } \\
\text { Genotypes }\end{array}$ & cases & MST & $\begin{array}{c}\text { Log-rank } \\
\text { P-value }\end{array}$ & Crude HR & $\mathbf{9 5 \%}$ CI (P-value) & aHR & 95\%CI(P-value) \\
\hline mir-149 rs2292832 & & & & & & \\
TT & 419 & 24.08 & 0.004 & 1 & & 1 & \\
CT & 120 & 28.50 & & 0.781 & $0.626-0.976(0.029)$ & 0.782 & $0.626-0.976(0.030)$ \\
CC & 45 & 32.59 & & 0.608 & $0.425-0.870(0.006)$ & 0.607 & $0.425-0.869(0.006)$ \\
TT & 419 & 24.08 & 0.002 & 1 & & 1 & \\
CC+CT & 165 & 29.62 & & 0.730 & $0.598-0.892(0.002)$ & 0.739 & $0.605-0.903(0.003)$ \\
CT+TT & 539 & 25.09 & 0.015 & 1 & & 1 & \\
CC & 45 & 32.59 & & 0.647 & $0.454-0.922(0.016)$ & 0.644 & $0.452-0.919(0.015)$ \\
mir-146a rs2910164 & & & & & & \\
CC & 189 & 25.68 & 0.983 & 1 & & 1 & \\
CG & 302 & 25.59 & & 0.986 & $0.807-1.204(0.886)$ & 0.999 & $0.818-1.221(0.995)$ \\
GG & 93 & 25.44 & & 1.005 & $0.766-1.319(0.969)$ & 0.967 & $0.737-1.269(0.808)$ \\
mir-196a2 rs11614913 & & & & & & \\
TT & 145 & 24.21 & 0.414 & 1 & & 1 & \\
CT & 319 & 26.07 & & 0.916 & $0.738-1.136(0.424)$ & 0.978 & $0.788-1.214(0.842)$ \\
CC & 120 & 27.17 & & 0.833 & $0.635-1.093(0.187)$ & 0.854 & $0.651-1.121(0.255)$ \\
mir-608 rs4919510 & & & & & & \\
GG & 154 & 24.79 & 0.803 & 1 & & & \\
CG & 308 & 23.53 & & 1.070 & $0.836-1.370(0.590)$ & 0.976 & $0.762-1.251(0.849)$ \\
CC & 122 & 25.06 & & 0.996 & $0.735-1.350(0.980)$ & 1.015 & $0.749-1.375(0.925)$ \\
mir-423 rs6505162 & & & & & & \\
CC & 386 & 24.27 & 0.874 & 1 & & & 1 \\
AC & 181 & 24.22 & & 1.010 & $0.805-1.269(0.929)$ & 1.017 & $0.809-1.277(0.886)$ \\
AA & 17 & 25.10 & & 0.853 & $0.453-1.606(0.622)$ & 0.953 & $0.506-1.796(0.881)$ \\
\hline
\end{tabular}

*MST: median survival time, aHR: HR adjusted by histological type, clinical stage, receipt of chemotherapy or surgery.

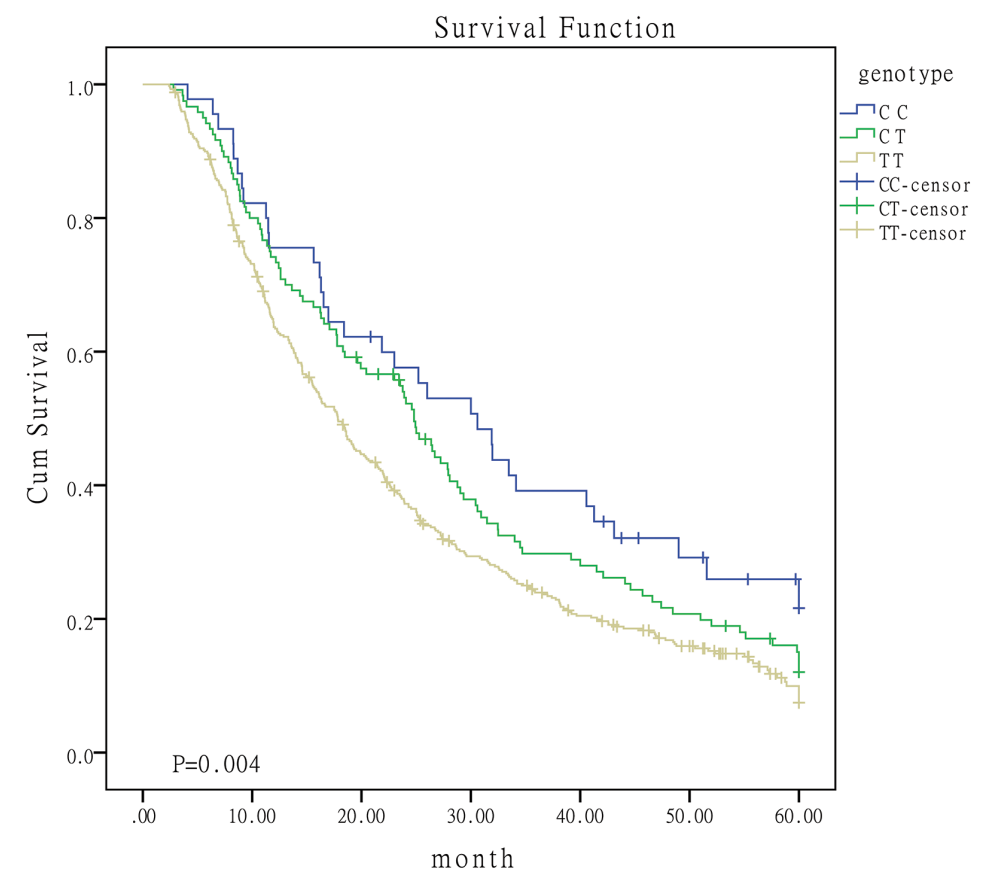

Figure 1: Survival curves for patients with different genotypes of miR-149. 
Table 3: Stratified analyses of the association between the five SNPs and OS

\begin{tabular}{|c|c|c|c|c|}
\hline SNPs subgroup & cases & Model & aHR & 95\%CI (P-value) \\
\hline \multicolumn{5}{|l|}{ mir-149 rs2292832 } \\
\hline \multirow[t]{3}{*}{ AGE $>60$} & 332 & CC vs TT & 0.494 & $0.294-0.830(0.008)$ \\
\hline & & $\mathrm{CC}+\mathrm{CT}$ vs TT & 0.693 & $0.509-0.943(0.020)$ \\
\hline & & $\mathrm{CC}$ vs $\mathrm{CT}+\mathrm{TT}$ & 0.519 & $0.311-0.866(0.012)$ \\
\hline \multirow[t]{2}{*}{$\mathrm{AGE} \leq 60$} & 252 & CT vs TT & 0.749 & $0.560-1.001(0.051)$ \\
\hline & & $\mathrm{CC}+\mathrm{CT}$ vs $\mathrm{TT}$ & 0.758 & $0.582-0.987(0.040)$ \\
\hline \multirow[t]{2}{*}{ Non-smoker } & 258 & CC vs TT & 0.376 & $0.205-0.691(0.002)$ \\
\hline & & CT vs TT & 0.537 & $0.376-0.767(0.001)$ \\
\hline Smoker & 326 & CC vs TT & 0.619 & $0.392-0.976(0.039)$ \\
\hline \multirow[t]{4}{*}{ SQU } & 230 & CC vs TT & 0.535 & $0.317-0.901(0.019)$ \\
\hline & & CT vs TT & 0.651 & $0.444-0.955(0.028)$ \\
\hline & & $\mathrm{CC}+\mathrm{CT}$ vs TT & 0.608 & $0.437-0.847(0.003)$ \\
\hline & & $\mathrm{CC}$ vs $\mathrm{CT}+\mathrm{TT}$ & 0.598 & $0.357-1.000(0.050)$ \\
\hline \multirow[t]{4}{*}{$\mathrm{I} / \mathrm{II} / \mathrm{III}$} & 509 & CC vs TT & 0.625 & $0.424-0.921(0.017)$ \\
\hline & & CT vs TT & 0.795 & $0.625-1.011(0.062)$ \\
\hline & & $\mathrm{CC}+\mathrm{CT}$ vs $\mathrm{TT}$ & 0.746 & $0.600-0.926(0.008)$ \\
\hline & & $\mathrm{CC}$ vs $\mathrm{CT}+\mathrm{TT}$ & 0.662 & $0.451-0.972(0.035)$ \\
\hline \multirow[t]{4}{*}{ Chemotherapy } & 529 & CC vs TT & 0.622 & $0.432-0.895(0.010)$ \\
\hline & & CT vs TT & 0.735 & $0.583-0.927(0.009)$ \\
\hline & & $\mathrm{CC}$ vs $\mathrm{CT}+\mathrm{TT}$ & 0.673 & $0.470-0.965(0.031)$ \\
\hline & & $\mathrm{CC}+\mathrm{CT}$ vs TT & 0.702 & $0.570-0.864(0.001)$ \\
\hline \multirow[t]{4}{*}{ Surgery } & 384 & CT vs TT & 0.677 & $0.509-0.901(0.008)$ \\
\hline & & $\mathrm{CC}$ vs TT & 0.540 & $0.348-0.839(0.006)$ \\
\hline & & $\mathrm{CC}+\mathrm{CT}$ vs TT & 0.635 & $0.492-0.819(<0.001)$ \\
\hline & & $\mathrm{CC}$ vs $\mathrm{CT}+\mathrm{TT}$ & 0.598 & $0.387-0.923(0.020)$ \\
\hline \multicolumn{5}{|c|}{ mir-196a2 rs11614913 } \\
\hline \multirow[t]{2}{*}{ SQU } & 230 & CC vs TT & 0.608 & $0.384-0.963(0.034)$ \\
\hline & & $\mathrm{CC}$ vs $\mathrm{CT}+\mathrm{TT}$ & 0.679 & $0.463-0.996(0.047)$ \\
\hline \multicolumn{5}{|l|}{ mir-608 rs4919510 } \\
\hline Chemotherapy & 529 & CC vs GG & 1.277 & $1.036-1.535(0.028)$ \\
\hline \multirow[t]{2}{*}{ Surgery } & 384 & CC vs GG & 1.411 & $1.066-1.814(0.040)$ \\
\hline & & $\mathrm{CC}+\mathrm{CG}$ vs $\mathrm{GG}$ & 1.359 & $1.056-1.762(0.013)$ \\
\hline
\end{tabular}

*SQU: squamous carcinoma, aHR: HR adjusted by histological type, clinical stage and therapeutic strategy. 
Table S2. The G-containing genotypes of miR-146a, C-containing genotypes of miR-149 and miR-196 were observed to be associated with increased expression level of mature miRNA. The association between the three SNPs and miRNA expression was described in Supplementary Figure S1. No significant association with miRNA expression was observed for miR-423 and miR608 SNPs.

The effect of rs2292832 on miR-149 expression was verified in our A549 cell lines. As shown in Supplementary Figure S2, vectors containing $\mathrm{C}$ allele has a higher expression level of miR-149 than those containing $\mathrm{T}$ allele. The expression level of vectors containing $\mathrm{C}$ allele was 1.55 times that of the vectors containing $\mathrm{T}$ allele $(\mathrm{P}<0.001)$.

The significant differences for cell proliferation was observed in our study. Results were shown in Figure 3. Vectors containing $\mathrm{C}$ allele may lead to decreased cell proliferation when compared to vectors containing $\mathrm{T}$ allele $(\mathrm{P}=0.038)$ and empty vectors $(\mathrm{P}<0.001)$. These results were accordant with the results in cell cycle analyses. As shown in Figure 4, cell number of G1 phase in vectors containing $\mathrm{C}$ allele was higher than that in vectors containing $\mathrm{T}$ allele $(\mathrm{P}=0.004)$ and empty vectors $(\mathrm{P}=0.023)$. Cell number of $\mathrm{S}$ phase in vectors containing $\mathrm{C}$ allele was lower than that in vectors containing $\mathrm{T}$ allele $(\mathrm{P}=0.001)$ and empty vectors $(\mathrm{P}=0.007)$. These results implied that cells containing $\mathrm{C}$ allele may obtain inhibition at $\mathrm{G} 1 / \mathrm{S}$ transition.

DNA topoisomerases IB (TOP1) was screened out as potential target mRNA of miR-149 by bioinformatics methods. Luciferase reporter assay was constructed to investigate the influence of miR-149 in activities of target gene. Results were shown in Figure 5. The relative luciferase activities for cells transfected with TOP13'UTR and miR-149 mimics were lower than the other three groups $(\mathrm{P}<0.001)$. We proceeded to perform the qRTPCR and western blotting to investigate the influence of miR-149 in trascription and translation of TOP1 gene. No significant effect on TOP1 mRNA levels was observed in

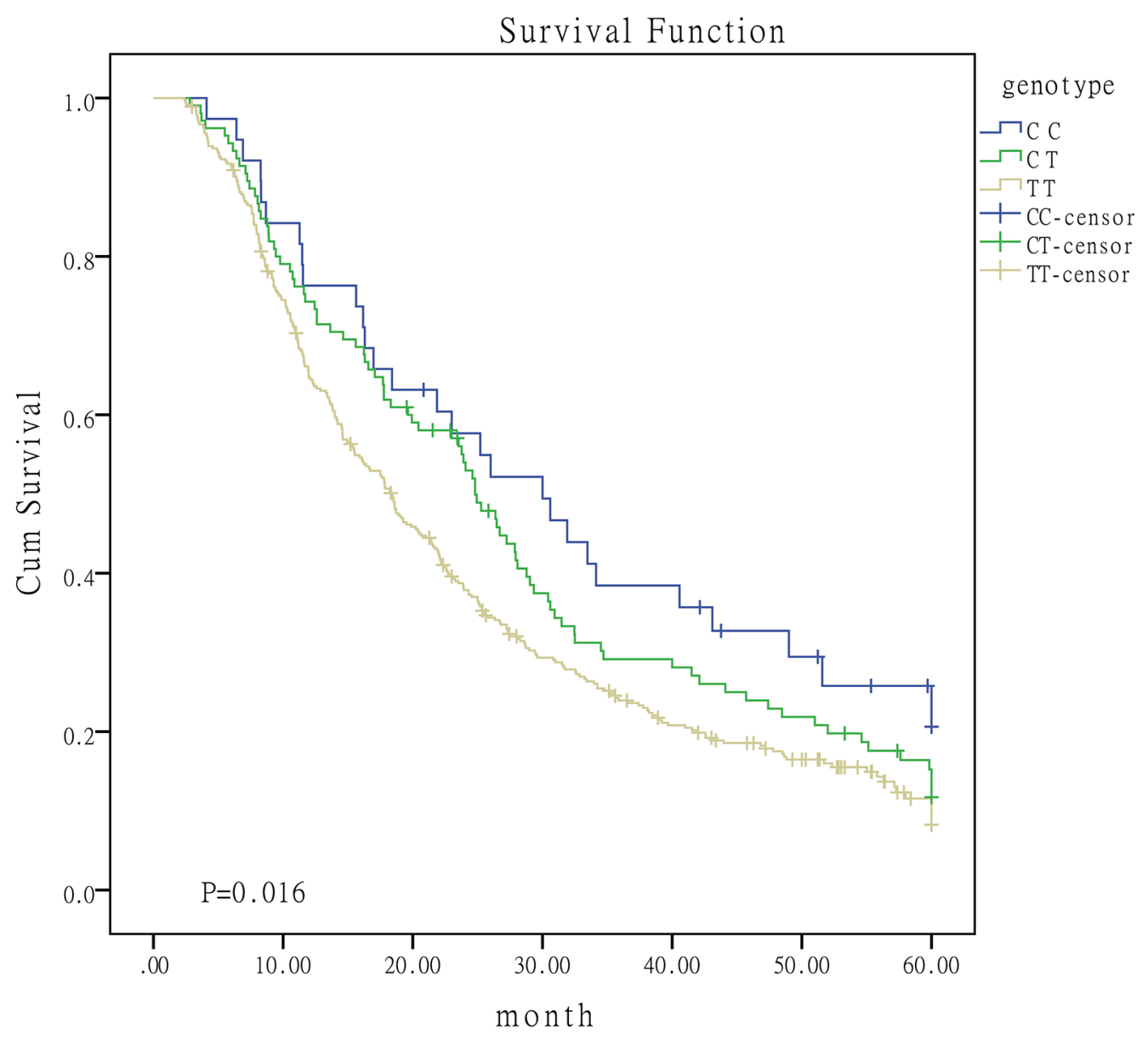

Figure 2: Survival curves for I/II/III patients with different genotypes of miR-149. 
Table 4: The joint effect of the miRNA SNPs on OS

\begin{tabular}{|c|c|c|c|c|c|c|}
\hline Strata & miRNAs & Model & Cases & HR & $95 \% \mathrm{CI}$ & $\mathbf{P}$ \\
\hline \multirow[t]{5}{*}{ SQU } & miR-149, miR-196a2 & & & & & \\
\hline & & $0^{*}$ & 42 & 1.00 & & \\
\hline & & $1^{*}$ & 98 & 0.886 & $0.594-1.323$ & 0.555 \\
\hline & & $2^{*}$ & 62 & 0.614 & $0.392-0.961$ & 0.033 \\
\hline & & $3+4^{*}$ & 28 & 0.484 & $0.270-0.866$ & 0.015 \\
\hline \multirow[t]{5}{*}{ Chemotherapy } & miR-149, miR-608 & & & & & \\
\hline & & $0^{\#}$ & 75 & 1.00 & & \\
\hline & & $1^{\#}$ & 229 & 1.097 & $0.787-1.529$ & 0.584 \\
\hline & & $2^{\#}$ & 168 & 1.029 & $0.733-1.445$ & 0.867 \\
\hline & & $3+4^{\#}$ & 57 & 0.572 & $0.367-0.893$ & 0.014 \\
\hline \multirow[t]{5}{*}{ Surgery } & miR-149, miR-608 & & & & & \\
\hline & & $0^{\#}$ & 57 & 1.00 & & \\
\hline & & $1^{\#}$ & 164 & 1.241 & $0.857-1.798$ & 0.253 \\
\hline & & $2^{\#}$ & 120 & 1.139 & $0.774-1.676$ & 0.509 \\
\hline & & $3+4^{\#}$ & 43 & 0.587 & $0.356-0.968$ & 0.037 \\
\hline
\end{tabular}

*0:TT of miR-149 and miR-196a2; 1:CT of miR-149 and TT of miR-196a2, or TT of miR-149 and CT of miR-196a2; 2: CT of miR-149 and miR-196a2, or CC of miR-149 and TT of miR-196a2, or TT of miR-149 and CC of miR-196a2; 3: CT of miR-149 and CC of miR-196a2, or CC of miR-149 and CT of miR-196a2; 4: CC of miR-149 and miR-196a2. \#0:TT of miR-149 and CC of miR-608; 1: TT of miR-149 and CG of miR-608, or CT of miR-149 and CC of miR-608; 2: TT of miR-149 and GG of miR-608, or CC of miR-149 and CC of miR-608, or CT of miR-149 and CG of miR-608; 3: CT of miR-149 and GG of miR-608, or CC of miR-149 and CG of miR-608; 4: CC of miR-149 and GG of miR-608 SQU:squamous cell carcinoma.

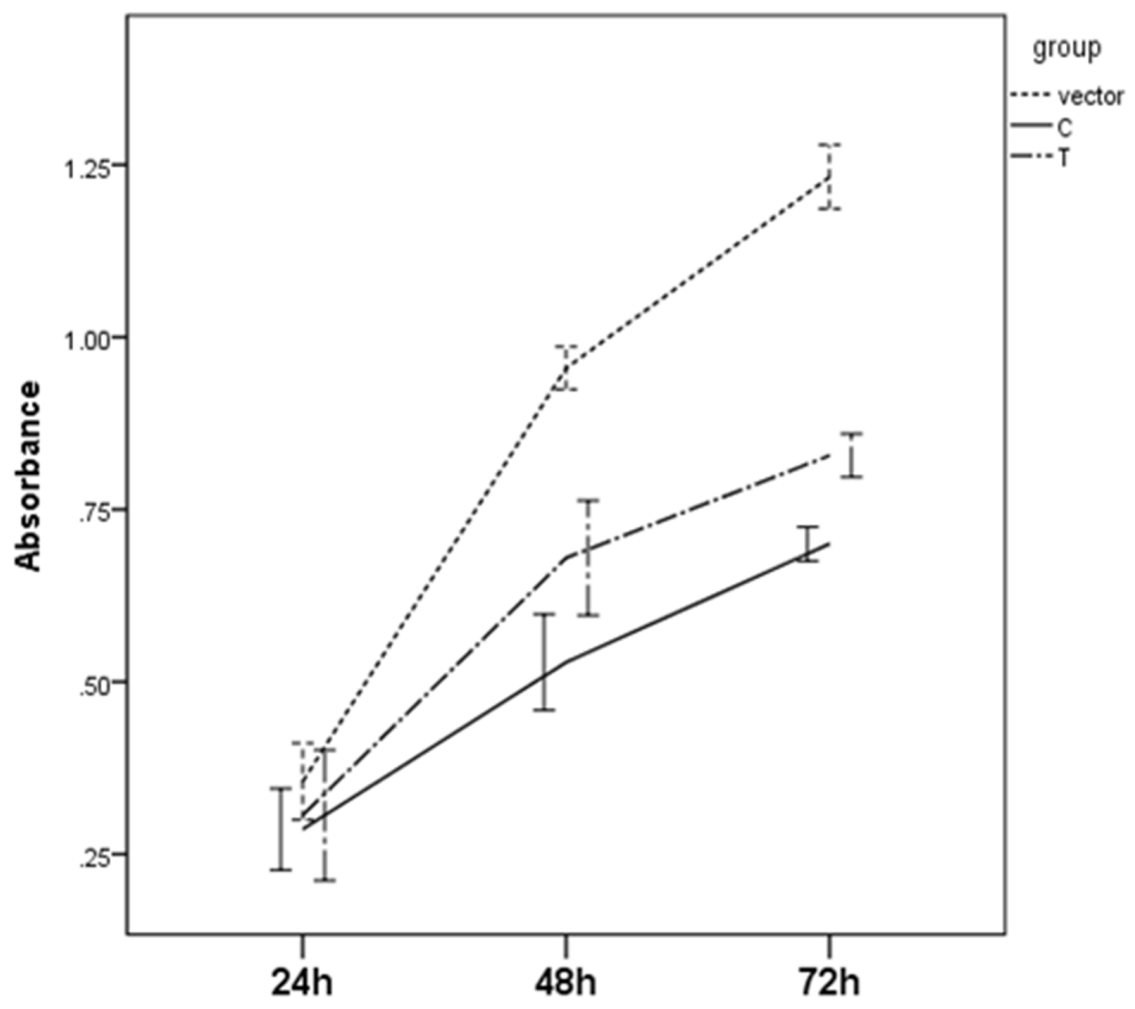

Figure 3: Effect of miR-149 rs2292832 on cell proliferation. 

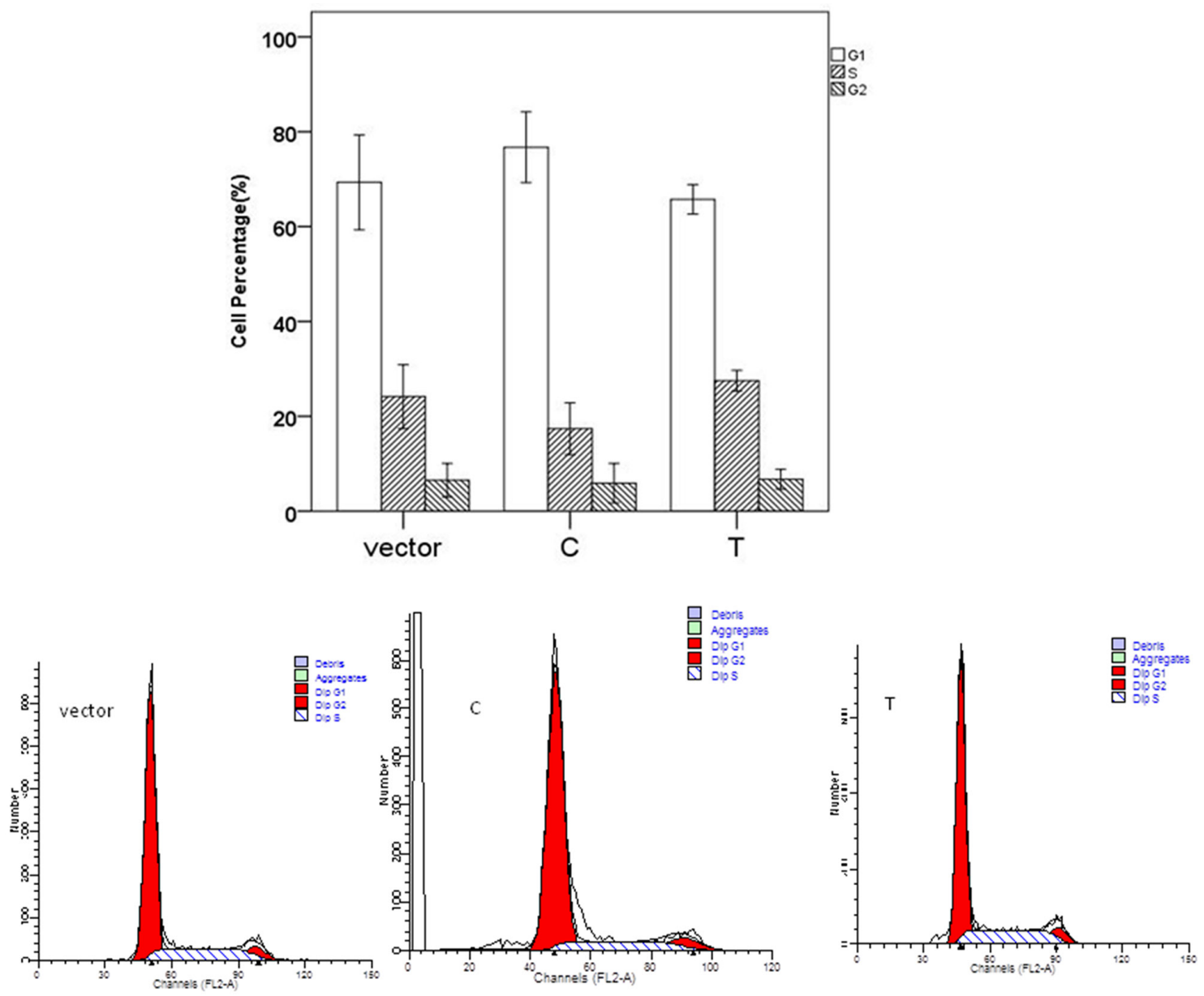

Figure 4: Effect of miR-149 rs2292832 on cell cycle.

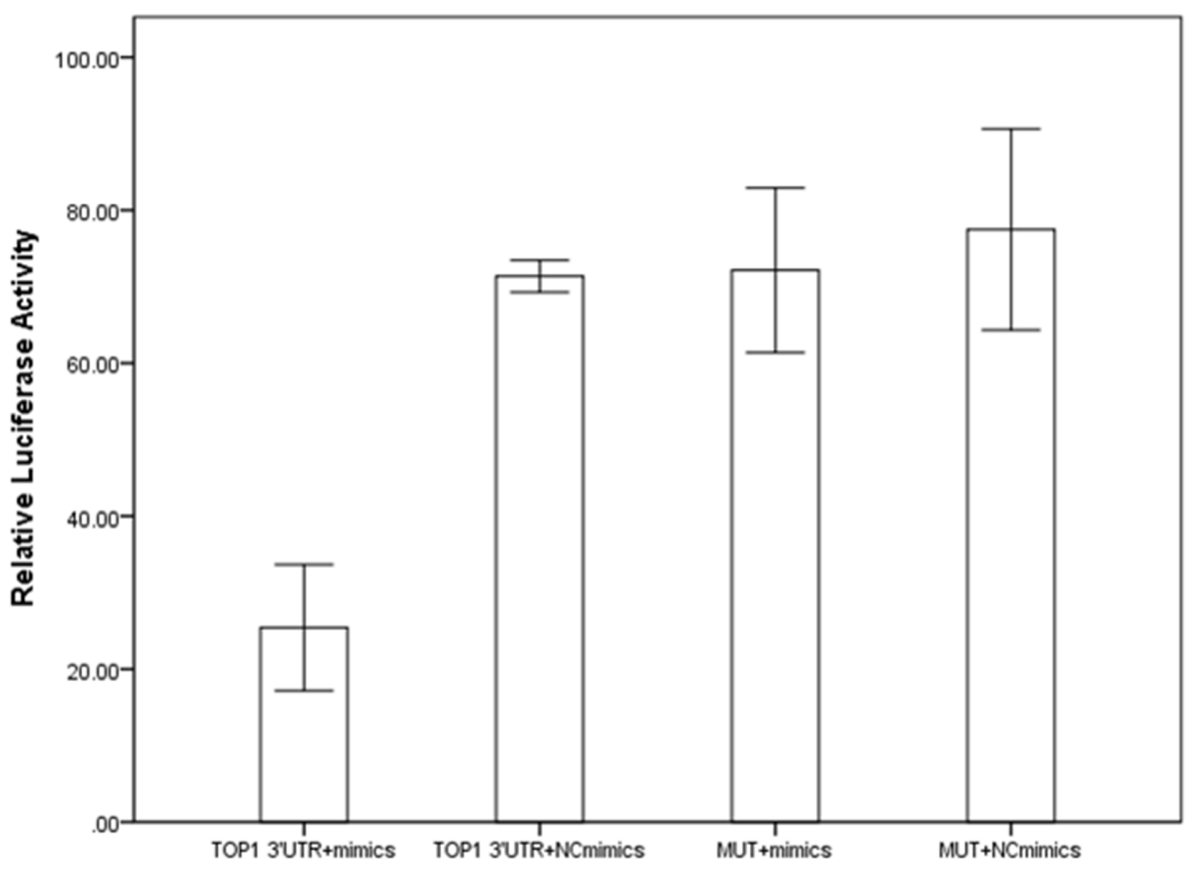

Figure 5: The results of luciferase reporter assay. 
qRT-PCR ( $\mathrm{P}=0.079$, Supplementary Figure S3). While, the significant effect on TOP1 protein expression was observed in western blotting $(\mathrm{P}<0.001$, Figure 6). These results implied that miR-149 SNP may influence the translation of TOP1 mRNA instead of the transcription of TOP1 gene.

miR-149 SNP conferred significant effect on survival rate for cells being treated with cisDichlorodiamineplatinum(II) (DDP) in our study. Results were shown in Figure 7. Being treated with concentrations of Rank 1, 5 and 6, cells containing $C$ allele were more sensitive to DDP than those containing $\mathrm{T}$ allele. Being treated with concentrations of Rank 2 and 7, cells containing $\mathrm{C}$ allele were less sensitive to DDP than those containing $\mathrm{T}$ allele.

\section{DISCUSSION}

The main results of our study is the protective effect of miR-149 SNPs on OS. Functional study results imply that $\mathrm{C}$ allele can increase the miR-149 expression levels, and therefore causing the decrease in TOP1 expression levels and cell growth. These results above explain the reasons that patients with $\mathrm{C}$ allele of miR-149 rs2292832 have better prognosis. We also observed the drug sensitivity for cells with C allele of miR-149 rs2292832 for certain DDP concentrations. These results support our observations that $\mathrm{C}$ allele is associated with better prognosis for patients who receiving chemotherapy.

The expression of miR-149 exhibited differently in cancer survival as previously reported [12-15]. Our results for miR-149 are consistent with Katada, Luo and Ke's studies [12-14]. Our study may help to have a deeper congnization of the role of miR-149 playing in cancer progression. Except for EMT and AKT1 signaling, miR-149 also involves in DNA topoisomerases functioning pathway to inhibit cancer cell growth. DNA topoisomerases are grouped as type I and type II. TOP1 (TOPIB) enzyme mainly involves in DNA relaxing to remove negative supercoils [16]. The inhibition of
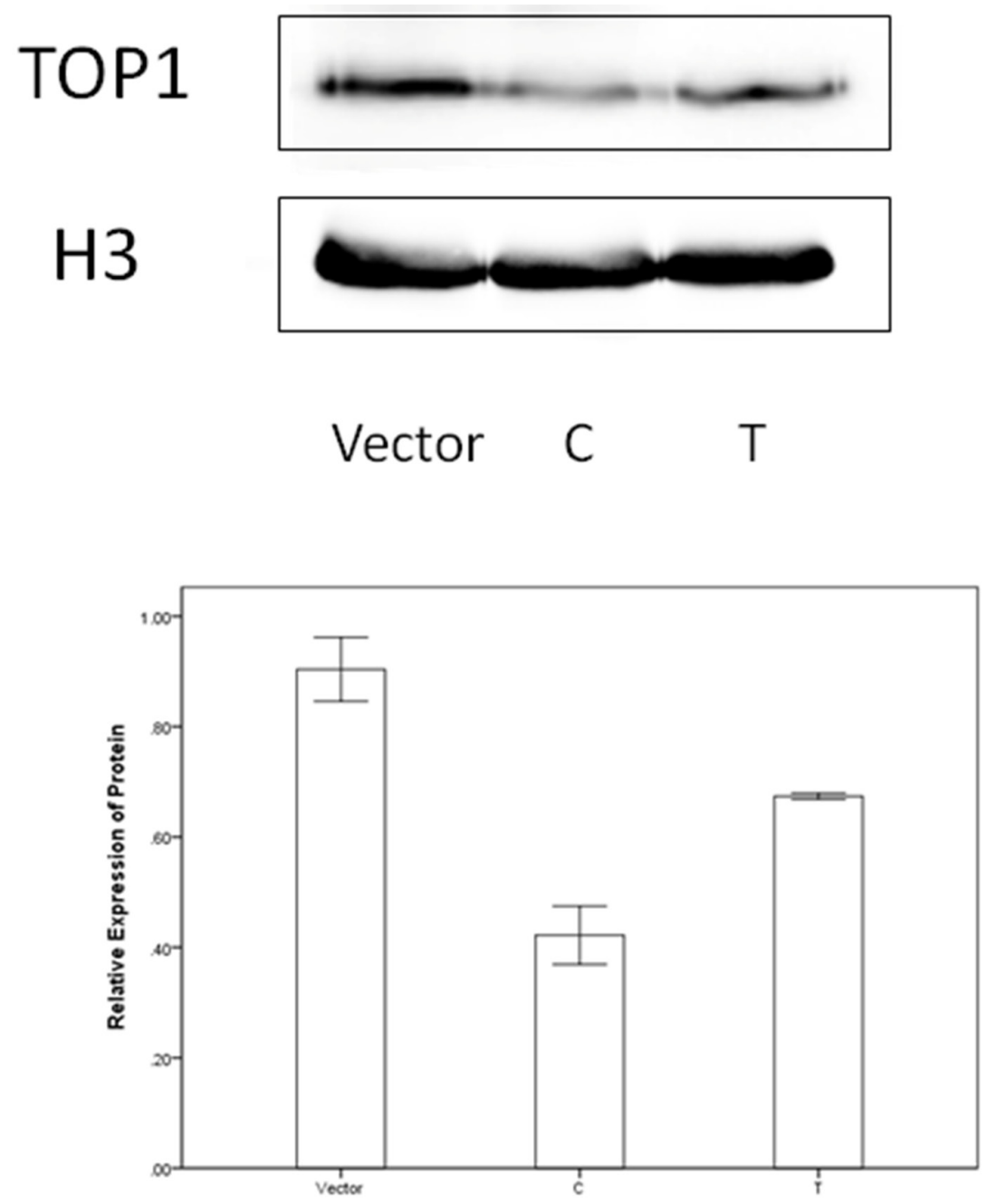

Figure 6: The western blotting results. 
TOP 1 expression may result in the increase in negative supercoils and therefore the decrease in cell growth. In our study, we observed the increase in miR-149 expression, the decrease in TOP1 protein levels and cell growth.

The prognostic role of miR-149 rs2292832 has been demonstrated previously in various types of cancer [17-24]. For NSCLC, results in our study are consistent with Hong's study [17]. For the previous study [17], the participants are early-stage, surgically resected NSCLC in Korea. While the subjects included in our study are 584 Chinese females with late-stage NSCLC. Another distinction from Hong's study is the association study for SNPs in miR-423 and miR-608 with NSCLC OS. Results in the present study support our previous study [25] and strengthen the role of miR-149 rs2292832 playing in NSCLC OS.

We also observed the significant association for miR-196a 2 and miR-608 with NSCLC OS. In previous study, we noticed that the CC genotype of miR-196a2 rs11614913 is a risk factor for II/IIIA NSCLC patients [17]. While, we cannot observe the same effect in our study. In our study, we only observed the protective role of
C-containing genotypes in squamous cell carcinoma. miR$608 \mathrm{CC}$ genotype showed the risk effect on OS for patients receiving chemotherapy. This may indicate that miR$608 \mathrm{CC}$ genotype may enhance the multi-drug resistant (MDR). While, the relatively small sample size for stratified analyses may shad the significance of the results. Larger studies are warranted to verify our results. The effect of miR-146a, miR-423 and their SNPs on cancer progression has been extensively studied previously [2632]. Nevertheless, the effect of the two genetic variants on NSCLC OS was not observed in our study.

Some defects exist in our study. Firstly, the sample size is not large in the cohort study. And the number is even smaller for stratified analyses. Secondly, only A549 cell lines were used in functional study. Thirdly, the function for miR-149 and its SNP was verified in vitro, without being verified in vivo.

In spite of the defects above, some advantages exist in our present study. One strength is that this is a multi-center study, enhancing the reliability of the results. Another one is the use of a systematic search for the genome-wide SNPs in miRNAs. The third one is that the

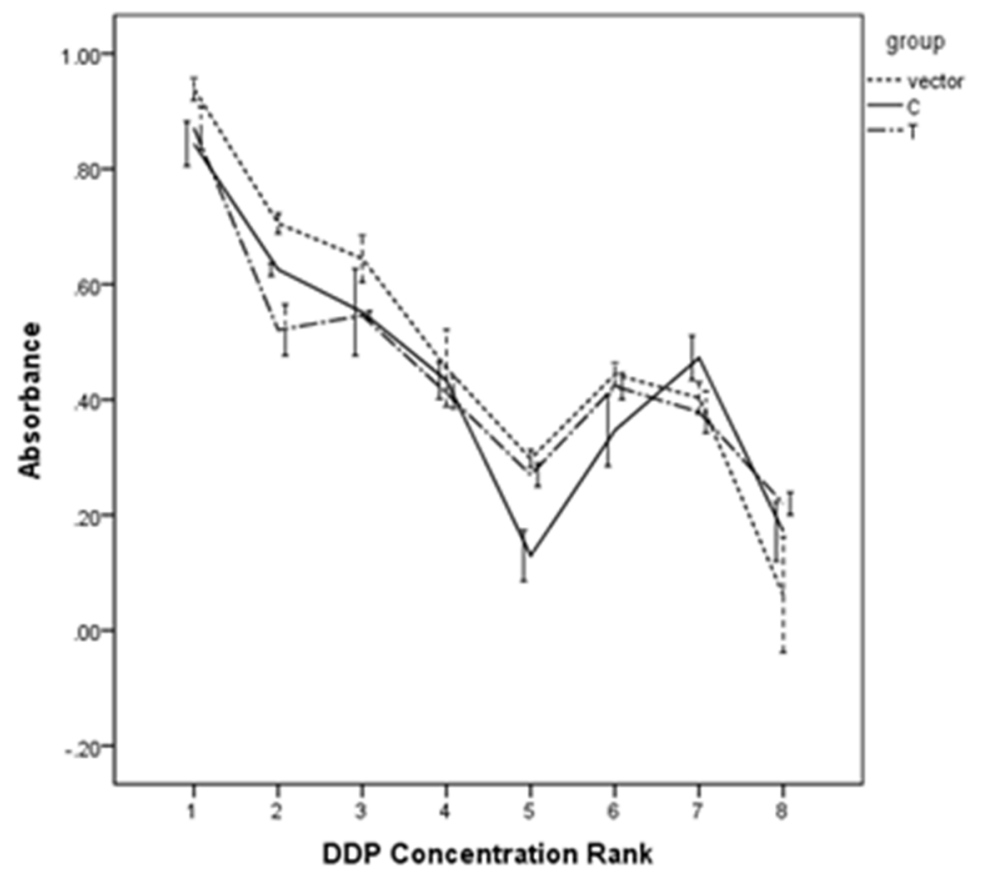

\begin{tabular}{lcccccccc}
\hline Rank & 1 & 2 & 3 & 4 & 5 & 6 & 7 & 8 \\
\hline Vectorvs C (P) & $<0.001$ & $<0.001$ & 0.001 & 0.200 & $<0.001$ & $<0.001$ & 0.001 & 0.002 \\
Vectorvs T (P) & 0.001 & $<0.001$ & 0.001 & 0.027 & 0.027 & 0.164 & 0.071 & $<0.001$ \\
Cvs T (P) & 0.045 & $<0.001$ & 0.732 & 0.192 & $<0.001$ & 0.001 & $<0.001$ & 0.066 \\
\hline
\end{tabular}

Figure 7: Resutls for drug sensitivity assays. 
subjects recruited are all females. Previous studies pay more attention to men and smokers. Although women were included in their studies, the number was relatively small. Our present study aimed to help to provide evidence for detection of prognostic biomarkers for females. The fourth one is the first time to obtain the role of miR-608 SNP in NSCLC OS, to the best of our knowledge. The last but not the least, this is the first report that TOP1 is the target mRNA of miR-149, to the best of our knowledge.

In conclusion, we provided evidence that miR149, miR-196a 2 and miR-608 polymorphisms may be associated with NSCLC overall survival in Chinese females. Our results support the prognostic role of miRNAs in cancer survival and the combination of miR149 with miR-196a 2 may act as a biomarker for squamous cell lung cancer prognosis. The certain combined genotypes of miR-149 and miR-608 SNPs can help to enhance the sensitivity to anti-cancer drugs and therefore predict a better OS. Our results are to be confirmed by larger prospective studies.

\section{MATERIALS AND METHODS}

\section{Study population}

This study was conducted in accordance with the amended Declaration of Helsinki. The institutional review board of China Medical University approved the protocol and written informed consent was obtained from all patients. All subjects were females and they were from unrelated ethnic Han Chinese. Estimates of the exposure to environmental factors were reported in previous study [33]. Individual with a total of 100 cigarettes in her lifetime was defined as a smoker, otherwise she was considered as a non-smoker [33]. The patients were recruited during March 2010 to March 2013 at the Liaoning Cancer Hospital \& Institute, Shenyang North Hospital and the fourth Affiliated Hospital of China Medical University. All patients were histologically confirmed when they were enrolled in the cohort. All subjects were interviewed and venous blood sample was obtained from each subject. Detailed background and clinical information including age, sex, tobacco exposure, TNM stage, clinical stage, histological type, receipt of chemotherapy and receipt of surgery has been collected.

Subjects received telephone follow-up every three months after being diagnosed with cancer. Data from Shenyang Center for Disease Control and Prevention (CDC) registry system were collected for death cause. Inpatient and outpatient medical records and Death Registry System of Shenyang Public Security Bureau were used to confirm the date of death. The patients were followed up to death or April 2015. Totally, we collected complete information of 584 patients. Another 74 patients were lost to follow up. In our study, the outcome event was defined as death from lung cancer. If not, the patients were considered survival no matter what the death cause was. The MST is 25.67 months in the ongoing study.

\section{SNP selection and genotyping}

We inquired the common hsa-miRNAs in public database miRBase (version 20.0) (Supplementary Table S3). We screened out 604 SNP sites with a MAF no less than $5 \%$ in Chinese Han population according to the data available in dbSNP. For these SNPs, we performed an extensive research in HapMap and Patrocles. 13 SNPs that located in pre-miRNA or mature miRNA with a MAF $>0.05$ were screened out. Given the consideration of false-positive rate by using a single bioinformatics tool, we return to miRBase and dbSNP to verify the sequences of the 13 SNPs. SNPs without being verified were excluded. We got 9 sites after above steps. Furthermore, the Gibbs binding free energy $(\Delta \mathrm{G}, \mathrm{Kcal} / \mathrm{mol})$ for each pair of common and variant alleles was computed using viennaRNA software [34]. The differences of the free energies between the two alleles were calculated as $\Delta \Delta \mathrm{G}$. We ranked the values of $\Delta \Delta \mathrm{G}$, and chose the $2 \mathrm{Kcal} /$ mol as cut-off. $3 \mathrm{SNPs}$ had a $\Delta \Delta \mathrm{G} \geq 2 \mathrm{Kcal} / \mathrm{mol}$. Among the SNPs with a $\Delta \Delta \mathrm{G}<2 \mathrm{Kcal} / \mathrm{mol}$, we found two SNPs located in two pivotal sites for miRNA maturation [10, 35]. rs6505162 located within 3nt of 3' pre-miRNA and rs4919510 located in dsRNA of pre-miRNA. Finally, we selected 5 SNPs (hsa-miR-196a2 rs11614913, hsa-miR146a rs2910164, hsa-miR-149 rs2292832, hsa-miR-423 rs6505162 and hsa-miR-608 rs4919510) (Supplementary Table S4). The work flow of SNP selection was exhibited in Supplementary Figure S4.

Phenol-chloroform method was utilized to extract genomic DNA from peripheral blood samples. The TaqMan allelic discrimination method was used to genotype the five selected SNPs according to the protocol. The samples were read and analyzed from the ABI 7500 Fast Sequence Detection System (Applied Biosystems, Lifetechnologies, USA). The average genotype call rate for all SNPs were $99.2 \%$. About $10 \%$ of the samples were randomly selected for confirmation by repeat genotyping, and the results were $100 \%$ concordant.

\section{Quantitative real time RT-PCR}

Sixty lung cancer tissues were obtained from surgically removed specimens of individual cases in this study (Supplementary Table S5). Total RNAs were extracted by Trizol reagent (Thermo, USA). TaqMan MicroRNA Reverse Transcription Kit (Applied Biosystem, USA) was utilized for cDNA synthesis. TaqMan MicroRNA Assays (Applied Biosystem, USA) was utilized to quantify mature miRNA expression levels according to the protocol. U6 was used as internal references. The PCR was repeated three times for every sample. Comparative CT method was used to evaluate the 
miRNA expression level. $2^{-\triangle \triangle \mathrm{CT}}$ is the relative miRNA expression.

\section{Cell lines and culture}

A549 cell lines were purchased from Shanghai Institute of Biochemistry and Cell Biology, Chinese Academy of Sciences (Shanghai, China). They were maintained in RPMI-1640 medium (Gibco, USA) with $10 \%$ fetal bovine serum (Gibco, USA), $100 \mathrm{U} / \mathrm{ml}$ penicillin sodium and $100 \mathrm{mg} / \mathrm{ml}$ streptomycin sulfate. Cell lines were cultured at $37^{\circ} \mathrm{C}$ with an air atmosphere containing 5\% carbon dioxide. In our experiment, cells were used and passaged when they reach the logarithmic phase of growth.

\section{Plasmid construction, lenti-virus production and stable transfection}

The common miR-149 expression construct (miR149-T) and the variant miR-149 expression construct (miR-149-C) were generated using GV369 vector (GeneChem, China). The sequence of both constructs were confirmed by direct sequencing. An empty GV369 vector was used as negative control. All the vectors were packaged with lentivirus and stable transfection were performed according to the manufacture's precedure. The transfection efficiency was assessed by counting green fluorescent protein-positive cells four days after the transfection. $2 \mathrm{ug} / \mathrm{ml}$ puromycin (Amresco, USA) were used to screen out the A549 cells transfected with miR149-T, miR-149-C and empty vectors.

\section{Cell proliferation and cell cycle}

To investigate the cell proliferation, cells were harvested $24 \mathrm{~h}, 48 \mathrm{~h}$ and $72 \mathrm{~h}$ after transfection. The survival rate were determined by using the Cell Counting Kit 8 (KeyGEN BioTECH, China). The absorbance value of each well was obtained at 450nm in the Bio-Rad M680 spectrophotometer (USA). Each time point and each experiment were repeated three times.

To investigate the cell cycle, cells were harvested $48 \mathrm{~h}$ after transfection and treated by using the Cell Cycle Detection Kit (KeyGEN BioTECH, China). DNA content was detected by flow cytometry on a FACS Calibur system (BD, USA). Results were exhibited as the percentage of cells in $\mathrm{G} 1, \mathrm{~S}$ and $\mathrm{G} 2$ phases.

\section{Drug sensitivity assay}

To investigate the drug sensitivity, cells were harvested $24 \mathrm{~h}$ after being treated with cisDichlorodiamineplatinum(II) (DDP) (Sigma, USA). According to the drug concentrations, cells were grouped as eight Ranks. For Rank 1, the drug concentration was $0.977 \mathrm{ug} / \mathrm{ml}$. For Rank 2, the drug concentration was 3.906ug/ml. For Rank 3, the drug concentration was $7.813 \mathrm{ug} / \mathrm{ml}$. For Rank 4, the drug concentration was $15.625 \mathrm{ug} / \mathrm{ml}$. For Rank 5, the drug concentration was $62.5 \mathrm{ug} / \mathrm{ml}$. For Rank 6, the drug concentration was $125 \mathrm{ug} /$ $\mathrm{ml}$. For Rank 7, the drug concentration was $250 \mathrm{ug} / \mathrm{ml}$. For Rank 8, the drug concentration was $500 \mathrm{ug} / \mathrm{ml}$. Cells treated with complete medium were used as negative controls.

\section{Identification of target mRNA of miR-149}

Starbase v2.0 was utilized to predict the potential target mRNAs of miR-149. Starbase includes the results from five informatics databases, TargetScan, PicTar, RNA22, PITA and miRanda/mirSVR (data shown in Supplementary Table S6). The potential mRNAs with at least two databases that indicating the target site were screened out. We proceeded to investigate the mature mRNA expression levels between lung cancer and normal tissues. The mRNAs with a fold change no less than 1.3 were screened out (Supplementary Table S7). Finally, we performed GO and KEGG analyses in String database for the mRNA of interest (Supplementary Table S8).

\section{RNA isolation from cell lines and qRT-PCR for detection of miR-149 and TOP1 mRNA}

Total RNAs were obtained with RNAiso Plus (KeyGEN BioTECH, China) following the manufacture's procedure. To detect the TOP1 mRNA expression levels, a PrimeScript RT reagent Kit with gDNA Eraser (Takara, Japan) was used for cDNA synthesis. SYBR Premix Ex Taq (Takara, Japan) was used for quantitative real time PCR and GAPDH was used as an internal control. The primers (Dingguo, China) for PCR were: TOP1-F: ACAGATGAGCAAGGAAGAG, TOP1-R: TGGAGGAGGAGAAGGAAC, Human GAPDHF258bp: AGAAGGCTGGGGCTCATTTG, Human GAPDH-R258bp:AGGGGCCATCCACAGTCTTC. To detect the miR-149 expression levels, TaqMan MicroRNA Reverse Transcription Kit (Applied Biosystems, USA) were used for cDNA synthesis and and TaqMan MicroRNA Assays (Applied Biosystems, USA) were used for quantitative PCR. U6 was used as an internal control. The PCR was repeated three times for every sample. The relative expression was calculated by the $2^{-\Delta \Delta C t}$ method.

\section{Western blotting}

Total proteins were isolated from nucleus by Nuclear and Cytoplasmic Protein Extraction Kit (Beyotime, China) and quantified using BCA method. Protein sample was analyzed on sodium dodecyl sulfate polyacrylamide gel electrophoresis $(10 \%$ seperation gel and $5 \%$ spacer gel). The antibodies were anti-TOP1, anti-H3 and anti-rabbit IgG (ProteinTech, China). The dilution ratio for anti-TOP1 was 1:2000. The dilution 
ratio for anti-H3 was 1:500. The dilution ratio for antirabbit IgG was 1:2000. The stripes were visualized by ECL methods (SuperSignal West Pico, Thermo, USA) and detected using Azure C300 biosystems (USA). The grey level values were read by Image J.

\section{Luciferase reporter assay}

TOP1 3'UTR fragment was inserted at the XbaI site, downstream of the luciferase gene in GV272 vector (GenChem, China) (Supplementary Figure S5). A vector containing the mutant of TOP1 3'UTR fragment was constructed as negative control. The vectors expressing ranilla luciferase were constructed as inner control. All of the sequences were confirmed by direct sequencing. Plasmids were isolated from E.coli by using TaKaRa MiniBEST Plasmid Purification Kit (Takara, Japan). The miR-149 mimics and the control miRNAs duplex (named as NC mimics) were purchased from GenePharma (China). The sequences were listed in Supplementary Table S9. Transfection was performed using Lipofectamine $3000 \mathrm{Kit}$ (Invitrogen) according to the manufacturer's procedure. Fourty-eight hours after the transfection, luciferase activities were measured using a Dual-Luciferase Reporter Assay System (Promega) in Synergy H1 systems (Bio Tek, USA). The relative luciferase activity was calculated as the ratio of firefly fluciferase value and ranilla luciferase value. The experiment was repeated three times.

\section{Statistical analysis}

Goodness-of-fit chi-square test was used to compare the death rates between groups with different baseline characteristics. The OS was calculated from the date at diagnosis to the date of last follow-up or death. Differences of MST between groups were estimated by log-rank test. Survival curve was estimated by Kaplan-Meier method and analyzed by the means of log-rank test. Univariate and multivariate Cox proportional hazards regression models were used to estimate the crude and adjusted hazards ratio (HRs) and their confidence interval (CIs). Student t-test and ANOVA were used to compare the differences for continuous variables. Statistically significant P-value is less than 0.05. All of the statistical analyses were performed in SPSS 17.0 and all P-values were two-sided.

\section{ACKNOWLEDGMENTS}

We sincerely thank for the help from Fu Weineng's team, Department of Medical Genetics, China Medical University, for offering advice on functional studies. We sincerely thank for the help from Qiu Xueshan's team, Department of Pathology, the First Affiliated Hospital and College of Basic Medical Sciences, China Medical University, for offering advice on cell culture. We sincerely thank for the contributions of every author in the study. XLZ designed the experiment and drafted the article, RYW, ZHB and ZYX included and excluded the participants, RYW, ZHB and ZYX collected the blood samples and conducted the follow-up, XLZ, YZH and LXL conducted the DNA extraction and genotyping, XLZ and LXL performed the functional studies, XLZ, LXL and RYW extracted and record the data, XLZ and YZH conducted the statistical analyses, YZH and LXL revised the article critically for important intellectual content, ZBS provided the fund and gave final approval of the version to be published.

\section{CONFLICTS OF INTEREST}

The authors declare no conflicts of interest.

\section{GRANT SUPPORT}

This study is supported by grant no.81272293 and no.81102194 from National Natural Science Foundation of China.

\section{REFERENCES}

1. Ferlay J, Soerjomataram I, Ervik M, Dikshit R, Eser S, Mathers C, Rebelo M, Parkin DM, Forman D, Bray F. GLOBOCAN 2012 V1.0, Cancer Incidence and Mortality Worldwide: IARC CancerBase No. 11. 2012.

2. Lyon F. International Agency for Research on Cancer. 2013:

3. Zeng HM, Zheng RS, Guo YM, Zhang SW, Zou XN, Wang N, Zhang LM, Tang JG, Chen JG, Wei KR, Huang SQ, Wang J, Yu L, et al. Cancer survival in China, 2003-2005. A population- based study. International Journal of Cancer. 2015: 136:1921-1930.

4. Liloglou T, Bediaga NG, Brown BR, Field JK, Davies MP. Epigenetic biomarkers in lung cancer. Cancer Lett. 2014: 342:200-212.

5. Ambros V. The functions of animal microRNAs. Nature. 2004: 431:350-355.

6. Zhou T, Zhang G-J, Zhou H, Xiao H-X, Li Y. Overexpression of microRNA-183 in human colorectal cancer and its clinical significance. European Journal of Gastroenterology \& Hepatology. 2014: 26:229-233.

7. Wang N, Li Y, Zhou R-M, Wang G-Y, Wang C-M, Chen Z-F, Liu W. Hsa-miR-196a2 functional SNP is associated with the risk of ESCC in individuals under 60 years old. Biomarkers. 2014: 19:43-48.

8. Chakraborty S, Mazumdar M, Mukherjee S, Bhattacharjee P, Adhikary A, Manna A, Chakraborty S, Khan P, Sen A, Das T. Restoration of $\mathrm{p} 53 / \mathrm{miR}-34 \mathrm{a}$ regulatory axis decreases survival advantage and ensures Bax-dependent apoptosis of non-small cell lung carcinoma cells. Febs Letters. 2014: 588:549-559.

9. Wu C, Li M, Hu C, Duan H. Prognostic role of microRNA polymorphisms in patients with advanced esophageal squamous cell carcinoma receiving platinum-based 
chemotherapy. Cancer Chemotherapy and Pharmacology. 2014: 73:335-341.

10. Lee Y, Ahn C, Han J, Choi H, Kim J, Yim J, Lee J, Provost P, Radmark O, Kim S, Kim N. The nuclear RNase III Drosha initiates microRNA processing. Nature. 2003: 425:415-419.

11. Chen J, Hu Z, Tian T, Zhou X, Miao R, Jin G, Ma H, Shen $\mathrm{H}$. Single nucleotide polymorphism associated with mature miR-196a2 influences the expression of Lymphocytespecific protein 1 (LSP1) gene. ACTA UNIVERSITATIS MEDICINALIS NANJING (Natural Science). 2009: 29:762-766.

12. Katada T, Ishiguro H, Kuwabara Y, Kimura M, Mitui A, Mori Y, Ogawa R, Harata K, Fujii Y. microRNA expression profile in undifferentiated gastric cancer. International Journal of Oncology. 2009: 34:537-542.

13. Luo Z, Zhang L, Li Z, Jiang C, Dai Y, Liu X, Zheng Y, Yu H, Xiang J, Li G. miR-149 promotes epithelial-mesenchymal transition and invasion in nasopharyngeal carcinoma cells. Journal of Central South University. Medical Sciences. 2011: 36:604-609.

14. Ke Y, Zhao W, Xiong J, Cao R. miR-149 Inhibits NonSmall-Cell Lung Cancer Cells EMT by Targeting FOXM1. Biochemistry research international. 2013: 2013:506731-506731.

15. Pan SJ, Zhan SK, Pei BG, Sun QF, Bian LG, Sun BM. MICRORNA-149 INHIBITS PROLIFERATION AND INVASION OF GLIOMA CELLS VIA BLOCKADE OF AKT1 SIGNALING. International Journal of Immunopathology and Pharmacology. 2012: 25:871-881.

16. Liu LF, Wang JC. Supercoiling of the DNA template during transcription. Proc Natl Acad Sci U S A. 1987: 84:7024-7027.

17. Hong M, Choi Y, Jang J, Jung H, Lee S, Lee W, Yoo S, Lee J, Cha S, Kim C, Lee E, Jeon H, Son J, et al. Association between Genetic Variants in Pre-MicroRNAs andSurvival of Early-Stage NSCLC. JOURNAL OF THORACIC ONCOLOGY. 2013: 8:703-710.

18. Hu Z, Chen J, Tian T, Zhou X, Gu H, Xu L, Zeng Y, Miao R, Jin G, Ma H, Chen Y, Shen H. Genetic variants of miRNA sequences and non-small cell lung cancer survival. J Clin Invest. 2008: 118:2600-2608.

19. Ahn DH, Rah H, Choi YK, Jeon YJ, Min KT, Kwack K, Hong SP, Hwang SG, Kim NK. Association of the miR$146 \mathrm{aC}>\mathrm{G}, \quad \operatorname{miR}-149 \mathrm{~T}>\mathrm{C}, \quad \mathrm{miR}-196 \mathrm{a} 2 \mathrm{~T}>\mathrm{C}$, and miR$499 \mathrm{~A}>\mathrm{G}$ polymorphisms with gastric cancer risk and survival in the korean population. Mol Carcinog. 2013: 52:39-51.

20. Kim WH, Min KT, Jeon YJ, Kwon CI, Ko KH, Park PW, Hong SP, Rim KS, Kwon SW, Hwang SG, Kim NK. Association study of microRNA polymorphisms with hepatocellular carcinoma in Korean population. Gene. 2012: 504:92-97.
21. Guan X, Sturgis EM, Song X, Liu Z, El-Naggar AK, Wei Q, Li G. Pre-microRNA variants predict HPV16positive tumors and survival in patients with squamous cell carcinoma of the oropharynx. Cancer Lett. 2013: 330:233-240.

22. Jang MJ, Kim JW, Min KT, Jeon YJ, Oh D, Kim NK. Prognostic significance of microRNA gene polymorphisms in patients with surgically resected colorectal cancer. Experimental and Therapeutic Medicine. 2011: 2:1127-1132.

23. Tu H, Liu C, Chang C, Wang P, Kao S, Yang C, Yu E, Lin S, Chang K. The Association between Genetic Polymorphism and the Processing Efficiency of miR-149 Affects the Prognosis of Patients with Head and Neck Squamous Cell Carcinoma. PLoS One. 2012: 7:e51606.

24. Zhang B. Single Nucleotide Polymorphisms of CASP-8 Genes, MicroRNA genes and Susceptibility and Prognosis of Malignant Lymphoma. Third Military Medical University. 2013.

25. Xia L, Ren Y, Fang X, Yin Z, Li X, Wu W, Guan P, Zhou B. Prognostic role of common microRNA polymorphisms in cancers: evidence from a meta-analysis. PLoS One. 2014: 9:e106799.

26. Yao Q, Cao Z, Tu C, Zhao Y, Liu H, Zhang S. MicroRNA146 a acts as a metastasis suppressor in gastric cancer by targeting WASF2. Cancer Lett. 2013: 335:219-224.

27. Chen G, Umelo IA, Lv S, Teugels E, Fostier K, Kronenberger P, Dewaele A, Sadones J, Geers C, De Greve J. miR-146a inhibits cell growth, cell migration and induces apoptosis in non-small cell lung cancer cells. PLoS One. 2013: 8:e60317.

28. Xu B, Wang N, Wang X, Tong N, Shao N, Tao J, Li P, Niu X, Feng N, Zhang L, Hua L, Wang Z, Chen M. MiR-146a suppresses tumor growth and progression by targeting EGFR pathway and in a p-ERK-dependent manner in castration-resistant prostate cancer. Prostate. 2012: 72:1171-1178.

29. Chaudhry MA, Omaruddin RA, Brumbaugh CD, Tariq MA, Pourmand N. Identification of radiation-induced microRNA transcriptome by next-generation massively parallel sequencing. J Radiat Res. 2013: 54:808-822.

30. Permuth-Wey J, Thompson RC, Burton Nabors L, Olson JJ, Browning JE, Madden MH, Ann Chen Y, Egan KM. A functional polymorphism in the pre-miR-146a gene is associated with risk and prognosis in adult glioma. $\mathrm{J}$ Neurooncol. 2011: 105:639-646.

31. Xing J, Wan S, Zhou F, Qu F, Li B, Myers RE, Fu X, Palazzo JP, He X, Chen Z, Yang H. Genetic polymorphisms in pre-microRNA genes as prognostic markers of colorectal cancer. cancer epidemiol biomarkers prev. 2012: 21:217-227.

32. Lin J, Horikawa Y, Tamboli P, Clague J, Wood CG, Wu $\mathrm{X}$. Genetic variations in microRNA-related genes are 
associated with survival and recurrence in patients with renal cell carcinoma. Carcinogenesis. 2010: 31:1805-1812.

33. Yin Z, Zhou B, He Q, Li M, Guan P, Li X, Cui Z, Xue X, Su M, Ma R, Bai W, Xia S, Jiang Y, et al. Association between polymorphisms in DNA repair genes and survival of nonsmoking female patients with lung adenocarcinoma. Bmc Cancer. 2009: 9:
34. Hofacker IL. Vienna RNA secondary structure server. Nucleic Acids Research. 2003: 31:3429-3431.

35. Bernstein E, Caudy AA, Hammond SM, Hannon GJ. Role for a bidentate ribonuclease in the initiation step of RNA interference. Nature. 2001: 409:295-296. 